Editorial, PET/CT Physics.

\title{
Machine Learning and Radiomics in Nuclear Medicine and Molecular imaging: Part I.
}

Magdy M. Khalil.

Medical Biophysics, Department of Physics, Faculty of Science, Helwan University.

\section{ABSTRACT:}

Diagnostic imaging modalities are undergoing a paradigm shift in terms of technological advances and their impact on many aspects of healthcare. The introduction of machine learning and radiomic in data analysis with capabilities of creating new clinical models has recently caught the attention of clinicians and scientists. Radiomics is a high throughput technology able to derive many imaging features from the diagnostic data while machine learning is a computer science discipline able to provide new forms of "electronic observer" able to mimic human tasks performed by radiologists and nuclear medicine physicians in daily routine. These technologies could be used individually or in combination to facilitate as well as solving issues associated with initial patient diagnosis, image processing, data analysis, stratification, prognosis and management. In the last decade, there was a rapidly growing interest in using radiomic in nuclear medicine and molecular imaging providing several solutions in reducing the injected radio activities, reducing imaging time, lesion segmentation, diagnosis, and many other applications that could potentially serve or replace current practices. The goal of this part of the machine learning and radiomics in nuclear medicine series is to introduce the reader to these new technologies and open avenues on current status, potential and future promises.

Key Words: Radiomics, Radio genomics, image segmentation, feature extraction, model validation. 


\section{INTRODUCTION:}

Nuclear medicine remains one of the most potential modalities in metabolic and molecular imaging (1). It has several quantitative capabilities that enable nuclear physicians and scientists to account for many biological and biochemical processes that occur during disease progression or regression. Lesion metabolic activity can be represented in different numerical forms in semi-quantitative or absolute quantitation. Recently, there is an increasing interest in deriving several texture features or statistical descriptors of the imaging data called radiomics in analogous to recent advances in the worlds of human genome "genomics", protein structure and functions "proteomics", biochemical metabolites "metabolomics" and other "omics" sciences ${ }^{(2-7)}$. Nuclear medicine was not an exception among imaging modalities in utilizing the power and application of radiomics in several malignancies. This type of data mining allows for deriving large amount of features that may account with variable extents on tumor heterogeneity providing not only phenotype-based characterization but also extended to comprise genotyping correlations ${ }^{(8)}$.
It is not only nuclear techniques that can be used in radiomic technology but also structural imaging modalities including $\mathrm{X}$-ray computed tomography (CT) and magnetic resonance imaging (MRI), ultrasound and pathological specimens/images are employed ${ }^{(2,9)}$.

The high throughput of these imaging features including data analysis and exploration to find out a relationship between pathology and imaging biomarkers was of particular interest among researchers. Precision medicine deals with optimizing individual patient management to reach the optimal decision making. Radiomics data could to large extent fit this purpose as it reveals quite large quantitative information about every particular imaging session.

However, the underlying statistical, physical and analytical methods need to be carefully reviewed in order to improve the clinical benefit as well as optimize research efforts. There are quite important steps that should be followed in order to successfully obtain meaningful results that can be correlated with patient physiologic or pathological status and generalize the model for better clinical decision making. 


\section{Egyptian J. Nucl. Med., Vol. 22, No. 1, June 2021}

This review and future releases will just place the reader on the essentials and milestones of the this rapidly growing field but was not meant to be extensive or exhaustive coverage of all recent approaches, methods and new developments. However, special focus will be placed on necessary parts and critical issues that face most researchers and investigators concerned with radiomics and radio genomics and their wide use applications.

Figure (1): describes the logical flow of most radiomics studies beginning from data acquision, processing and segmentation, feature extraction, model development and validation.

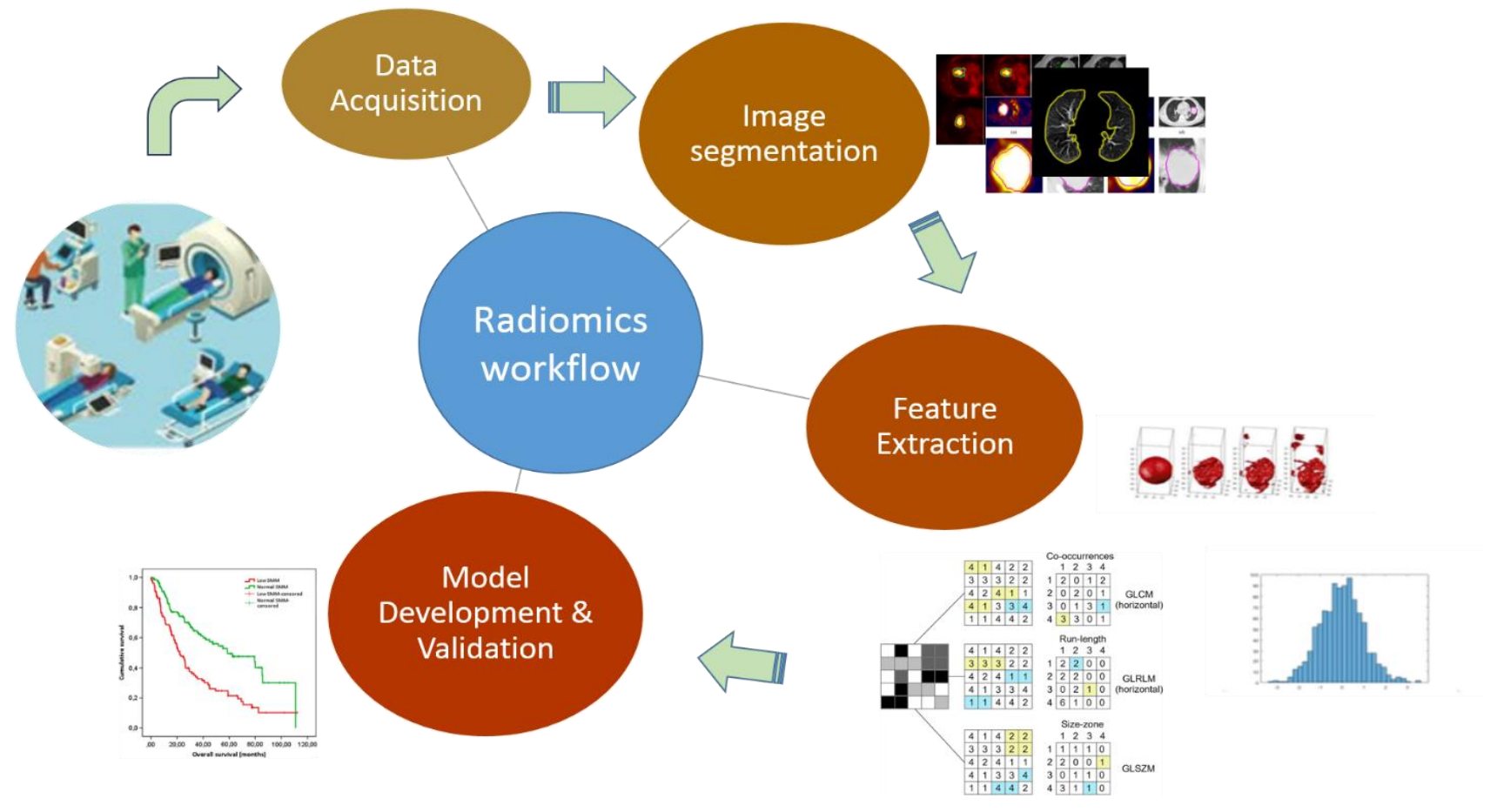

Figure (1): Radiomics workflow including several steps of image acquision, processing, segmentation, feature extraction, model development and validation. 


\section{Lesion segmentation:}

Initial steps that are normally taken to implement radiomics data analysis are to extract the lesion out of the surrounding organ or structures using region or volume of interest (ROI/VOI). It represents which part of the image that needs to be selected for further analysis. It can be segmented manually or (semi-) automatically ${ }^{(10)}$. The process of tumor delineation can introduce undesired bias into data since segmentation methods vary widely and hence results are subject to fluctuations that might affect reproducibility and hence clinical model instability ${ }^{(\mathbf{1 0})}$. Manual segmentation as recommend by experts in the filed should be avoided as it introduce variability with reduced agreement among observers. Threshold based methods seem to have also lower performance while semi-automated methods with threshold was found to offer higher reproducibility among different observers. The key point is how the segmentation is implemented and how is it sensitive and robust to radiomic analysis. A recent report has emphasized and argued the use of PET radiomics mainly due to impact of contouring variability on PET-based radiomics that hinder its translation among different institutions ${ }^{(11)}$.

\section{Pixel/Voxel size:}

While pixel or voxel size is determined prior to image acquision or during image reconstruction based on system resolving capabilities, radiomics analysis was shown to be affected by selection of the pixel size and whether isotropic or not-isotropic. This has been widely investigated in CT dataset and was shown to impact reproducibility of some features ${ }^{(11)}$. Normalizing those features to voxel size was shown to eliminate variability's among measurements. Two approaches are present to harmonize voxel size including image reconstruction and voxel interpolation. The former mightn't be easy accessible as raw data in some instances are not available whereas the former introduce some information that could confound the results ${ }^{(10}$, 12). Reconstruction was shown to reduce bias introduced in the PET images when 3D PET was compared to 4D PET data. It seems that there is room for further research on how and which voxel size is optimally selected in PET radiomics. 


\section{Intensity Discretization:}

The range of SUV values that may a particular patient has in one; two or more lesions may vary substantially in the intensity levels with sparsely of the lesion histogram. In order to standardize the analysis and feature matrix calculations, the use of intensity level discretization appeared to be an important step to maintain consistency of the results ${ }^{(\mathbf{1 3})}$. The two most common methods of intensity discretization are the bin number and bin width. Both of the two approaches have their weaknesses and strengths. The former is employed when dividing the SUV range into equally spaced bins, where the intensity values (bin size) varies per image keeping a constant intensity values per resolution bin. This is collectively called discretization level parameter which was found to impact reliability of the measurements ${ }^{(13,14)}$.

\section{Processing software's:}

There are many software packages that are available to calculate radiomic feature for PET, SPECT, CT and MR Datasets. The variability among those codes would introduce another source of variation that was initially considered in the development of imaging biomarker standard initiative (IBSI) accounting for less than 25\% taking into account standardization of the processing steps ${ }^{(\mathbf{1 2})}$. This could be enhanced to get rid of the choice of which software program would be utilized as well as elimination of the intervariation among radiomics data analysis. Many software programs and codes along with their functionalities and features are described in the literature where some of them can be applied via internet online ${ }^{(\mathbf{1 5})}$.

\section{Data generation and Phantom studies:}

Analyzing radiomic data obtained from more than one scanner might subject to interscanner variations and thus a sort of removing the effect of particular system characteristics from the data is necessarily required to remove probable data bias. Phantom examinations have been extensively applied in medical imaging research but could be an acceptable alternative in determination of feature reproducibility, robustness, repeatability as well as stability ${ }^{(16)}$. The above mentioned variations could also be assessed using phantom experiments. However, the biological relevance and variations induced by individual patient biological characteristics hinder from deducing or creating a successful or reliable clinical decision models. 


\section{Type of Features and Radiomics}

\section{Descriptors:}

\section{Morphological Descriptors.}

Morphological or shape-based descriptors serve to describe the geometric shape or surface of the region of interest such as diameters, eccentricity, short and long axis as well as surface and volume or their ratio. Other morphological features are compactness circularity, elongation, roughness, eccentricity, extent, solidity, and sphericity, convexity, aspect ratio. These features are generally easy to implement and has been used in several biomedical imaging data mining with recent development in 3D printing ${ }^{(16)}$.

\section{Gradient based methods:}

The gray level transition from voxel to another could be a useful measure of tumor heterogeneity such as an abrupt change from black to white or the reverse ${ }^{(6)}$.

This could be used as an indication or statistical descriptors to differentiate among tumor phenotypes. It might include gradient mean, gradient variance, kurtosis and skewness. Skewness is a measure of the histogram symmetry while kurtosis is a measure of the peak-endless of the histogram intensity curve.

\section{First order: Histogram Features.}

Histogram based features extract relies on a global measure of lesion gray level intensity including minimum, maximum, mean, standard deviation, percentiles, variance and others. The conventional PET metrics that normally used in the clinic also belong to this first order features that include SUV max, SUV mean, SUV peak. However, there are other measures such as mean, maximum, median, variance, histogram entropy and uniformity/energy that might be of particular interest.

\section{Gray-Level Co-occurrence Matrix:}

(GLCM).

Gray-level co-occurrence matrix looks at the spatial arrangement of each pair of voxels with prior definition of their intensity levels including different directions and can be applied to 2D as well as 3D datasets ${ }^{(\mathbf{1 7})}$. The former can be measured in horizontal, vertical and diagonal while a number of 13 directions can be applied to 3D data. Entropy is a GLCM member and describes the randomness or inhomogeneity of gray levels within a given region. Other metrics of this matrix include angular second moment and contrast. The angular second moment refers to intensity homogeneity while contrast deals with graylevel differences between voxels or voxel pair. 


\section{Gray-Level Run-length Matrix (GLRLM):}

The GLRLM matrix cares with connected the spatial arrangements of connected voxels that run with the same level of intensity for 2 or more directions ${ }^{(5)}$. In other words, the spatial distribution of pixel values is recorded by counting the lengths of consecutive runs that have the same intensity level. The features of this matrix include short run emphasis, gray level and run-length non-uniformity, fraction and others. The fraction reflects the graininess as it accounts for percent of voxels that are part of the runs. The short and long run emphasis are weighted measure of the length of the runs being penalized by the length of the run.

\section{Gray-Level Size Zone Matrix (GLSZM):}

The GLSZM is similar to the GLRLM. However, it deals with connected voxels or number of groups (i.e. zones) having the same intensity values not necessarily for different directions. The voxels selected may be measured at distances that define neighborhood. The GLSZM computes intensity level zones in an image ${ }^{\left({ }^{(6)}\right.}$. The intensity or gray level zone is defined as the number of connected voxels that share the level of intensity.
A voxel is considered connected if the distance is 1 according to the criteria of 26connected region in $3 \mathrm{D}$ or 8 -connected region in 2D. Small Area Emphasis (SAE), Large Area Emphasis (LAE), and Gray Level NonUniformity (GLN) are among the different measures of the GLSZM.

Another similar matrix is the Gray-Level Distance Zone Matrix (GLDZM). It doesn't only compute connected voxel zones with the same gray level but requires those voxels to be at the same distance from the ROI edge. The GLDZM is a combination between lesion morphology and texture features. The small distance high gray level emphasis is one of the features of the GLDZM.

\section{Neighborhood Gray-Tone Difference}

\section{Matrix (NGTDM):}

The NGTDM matrices are higher order texture and serve to assess the variation between a voxel intensity value and the mean intensity level of the neighboring voxels within a certain distance ${ }^{(6)}$. Some of the key features of the NGTDM matrix are complexity, coarseness, and busyness. Coarseness quantifies the rate of spatial change in intensity levels such that lower rate of intensity changes indicates greater coarseness. 
Busyness feature is used to assess the rapid changes of the central voxel with respect to neighboring ones. High coarseness in a VOI reflects small areas with high fluctuating intensity of voxel values. In case of one voxel a matrix of 8 neighboring voxel is formed in an array of $3 \times 3$. The matrix then measures the sum of absolute difference that exists between the centered voxel and the neighboring ones (5)

\section{Neighborhood Gray-Level Dependence Matrix (NGLDM):}

As the name implies, the NGLDM matrix assess a central voxel with the respect with its neighboring voxels. The distance of the neighboring voxels is controlled through a predefined difference value (i.e. intensity values) between the central voxel and the neighboring voxels. It records the difference of grey-level between one voxel and its 26 neighbors in 3 dimensions (8 in 2D). Similarly, as described in previous matrices, NGLDM_ coarseness is the level of spatial rate of change in intensity, NGLDM_ contrast is the intensity difference between neighboring regions and NGLDM busyness is the spatial frequency of changes in intensity ${ }^{(18)}$.

\section{Issues of Radiomics data analysis:}

The field of radiomic and radio genomics was received with enthusiasm and potential but posed challenges and limitations that must be seriously tackled including limited datasets for evaluations (i.e. number of patients), feature selection, large features dimensions "curse of dimensionality", and most importantly is standardization of the processing steps and harmonization among clinical centers.

The use of artificial intelligence and more specifically deep learning was also an important element in texture feature extraction and development of computer assisted software program able to aid physicians in several tasks including lesion contouring, segmentation, diagnosis, prognosis and other clinically relevant tasks. 


\section{REFERENCES:}

1. Khalil, MM (ed). Basic Science of PET Imaging. Springer; 2017.

\section{Aerts HJ, Velazquez ER, Leijenaar RT, et} al. Decoding tumour phenotype by noninvasive imaging using a quantitative radiomics approach. Nat. Commun.5. 4006; 2014.

3. Chen B, Yang L, Zhang $R$, et al. Radiomics: an overview in lung cancer management-a narrative review. Ann. Transl. Med. 8(18); 1191; 2020.

\section{Edalat-Javid M, Shiri I, Hajianfar G, et} al. Cardiac SPECT radiomic features repeatability and reproducibility: A multiscanner phantom study. J. Nucl. Cardiol; 2020.

5. Ha S, Choi H, Paeng JC, et al Radiomics in Oncological PET/CT: a Methodological Overview. Nucl. Med. Mol. Imaging. 53 (1): 14-29; 2019.

6. Mayerhoefer ME, Materka A, Langs $G$, et al. Introduction to Radiomics. J. Nucl. Med. 61(4):488-95; 2020.
7. Song J, Yin Y, Wang $H$, et al. A review of original articles published in the emerging field of radiomics: Eur. J. Radiol. 127:108991; 2020.

8. Wu J, Tha KK, Xing L, et al. Radiomics and radiogenomics for precision radiotherapy. J. Radiat. Res. 59(suppl_1): i25-i31; 2018.

9. Wu Q, Wang $S$, Chen $X$, et al. Radiomics analysis of magnetic resonance imaging improves diagnostic performance of lymph node metastasis in patients with cervical cancer. Radiother. Oncol.138:141-8; 2019.

10. Zwanenburg A. Radiomics in nuclear medicine: robustness, reproducibility, standardization, and how to avoid data analysis traps and replication crisis. Eur. J. Nucl. Med. Mol. Imaging: 46(13):2638-55; 2019.

11. Yang F, Simpson G, Young L, et al. Impact of contouring variability on oncological PET radiomics features in the lung. Sci. Rep.10(1): 369; 2020. 
12. Zwanenburg A, Vallieres M, Abdalah MA, et al. The Image Biomarker Standardization Initiative: Standardized Quantitative Radiomics for High-Throughput Image-based Phenotyping.

Radiology; 295(2):328-38; 2020.

\section{Leijenaar RT, Nalbantov G, Carvalho $S$,} et al. The effect of SUV discretization in quantitative FDG-PET Radiomics: the need for standardized methodology in tumor texture analysis; Sci. Rep. 5:11075; 2015.

\section{Liberini V, De Santi B, Rampado O, et al.}

Impact of segmentation and discretization on radiomic features in (68)Ga-DOTA-TOC $\mathrm{PET} / \mathrm{CT}$ images of neuroendocrine tumor. EJNMMI Phys. 8(1): 21; 2021.
15. Yuan R, Shi S, Chen J, et al. Radiomics in RayPlus: a Web-Based Tool for Texture Analysis in Medical Images. J. Digit. Imaging: 32(2):269-75; 2019.

16. Limkin EJ, Reuze S, Carre A, et al. The complexity of tumor shape, spiculatedness, correlates with tumor radiomic shape features: Sci. Rep. 9(1):4329; 2019.

\section{Haralick $R M$ and Shanmugam $K$.} Textural features for image classification. IEEE. Trans. Syst. Man. Cybern. SMC-3:610$621 ; 1973$.

18. https://www.lifexsoft.org. Accessed 2nd May; 2021. 